\title{
Philosophiques
}

\section{Pas de course. Notes piquées sur le séminaire « GREPH. Le concept de l'idéologie chez les idéologues français » (1974-1975)}

\section{Thomas Clément Mercier}

Volume 47, numéro 2, automne 2020

Derrida en cours

URI : https://id.erudit.org/iderudit/1075125ar

DOI : https://doi.org/10.7202/1075125ar

Aller au sommaire du numéro

Éditeur(s)

Société de philosophie du Québec

ISSN

0316-2923 (imprimé)

1492-1391 (numérique)

Découvrir la revue

Citer cet article

Mercier, T. C. (2020). Pas de course. Notes piquées sur le séminaire « GREPH. Le concept de l'idéologie chez les idéologues français » (1974-1975).

Philosophiques, 47(2), 289-313. https://doi.org/10.7202/1075125ar
Résumé de l'article

Afin d'examiner la forme et la fonction « cours » dans le corpus de Derrida, je propose une lecture du séminaire inédit « GREPH. Le concept de l’idéologie chez les idéologues français » (1974-1975). Ce séminaire fut mené dans le cadre des engagements politico-institutionnels du GREPH (Groupe de recherches sur l'enseignement philosophique). Il constitue donc un point d'entrée privilégié pour saisir le rapport complexe que Derrida entretient avec l'enseignement, et plus généralement avec l'institution philosophique. Au cours de ce séminaire, Derrida s'intéresse au « corps enseignant » en tant qu'il fonctionne comme " machine reproductrice » au sein de l'organisation économique générale de la société. Courant deux lièvres à la fois, Derrida mêle une réflexion généalogique sur l'histoire de l'enseignement philosophique en France et en Europe à une analyse théorique de concepts marxistes et sociologiques tels que " capital ", " reproduction », ou " appareil idéologique ». À travers des lectures déconstructrices et transformatrices de Marx et d'Althusser, Derrida s'efforce de penser une autre économie - une économie du texte débordant la logique reproductrice qu'il identifie aussi bien chez les économistes classiques que chez leurs critiques marxistes. 


\title{
Pas de course. Notes piquées sur le séminaire «GREPH. Le concept de l'idéologie chez les idéologues français » (1974-1975)
}

\author{
THOMAS CLÉMENT MERCIER \\ ANID/FONDECYT / Universidad Adolfo Ibañez (Santiago, Chile)
}

\begin{abstract}
RÉSUMÉ. - Afin d'examiner la forme et la fonction «cours » dans le corpus de Derrida, je propose une lecture du séminaire inédit «GREPH. Le concept de l'idéologie chez les idéologues français» (1974-1975). Ce séminaire fut mené dans le cadre des engagements politico-institutionnels du GREPH (Groupe de recherches sur l'enseignement philosophique). Il constitue donc un point d'entrée privilégié pour saisir le rapport complexe que Derrida entretient avec l'enseignement, et plus généralement avec l'institution philosophique. Au cours de ce séminaire, Derrida s'intéresse au «corps enseignant» en tant qu'il fonctionne comme «machine reproductrice» au sein de l'organisation économique générale de la société. Courant deux lièvres à la fois, Derrida mêle une réflexion généalogique sur l'histoire de l'enseignement philosophique en France et en Europe à une analyse théorique de concepts marxistes et sociologiques tels que «capital», «reproduction», ou «appareil idéologique». À travers des lectures déconstructrices et transformatrices de Marx et d'Althusser, Derrida s'efforce de penser une autre économie - une économie du texte débordant la logique reproductrice qu'il identifie aussi bien chez les économistes classiques que chez leurs critiques marxistes.
\end{abstract}

\begin{abstract}
In order to examine the form and function of the course in Derrida's corpus, I propose a reading of the unpublished seminar "GREPH. Le concept de l'idéologie chez les idéologues français" (1974-1975). This seminar was given as part of Derrida's political and institutional engagements within GREPH (Groupe de recherches sur l'enseignement philosophique). As such it offers many insights into Derrida's complex relationship with teaching and more broadly with the institution of philosophy. In the course of the seminar, Derrida examines the "teaching body" inasmuch as it functions as a "reproductive machine" within the general economic organization of society. Running on two different tracks at once, Derrida combines a genealogical study of the history of the teaching of philosophy in France and Europe with a theoretical analysis of Marxist and sociological concepts such as "capital", "reproduction", and "ideological apparatus". Through deconstructive-transformative readings of Marx and Althusser, Derrida proposes to think another economy - an economy of the text exceeding the reproductive logic which he discerns in classical economists as well as in their Marxist critics.
\end{abstract}


Il paraît heureux en séminaire, du moins je le crois. C'est donc qu'il rêve, et en rêve parle en poète ce dont il voit la foule témoigner. Il serait même heureux, j'en suis sûre, si le cours de son séminaire n'était pas toujours si court. Il se demande toujours pourquoi le cours doit être court. Pourtant le cours d'un fleuve, se dit-il, en débordant l'arrêt de course du cours

(Ici commencerait une longue course autour du mot cours. Cours! Mais il faut, c'est l'édit de ce livre, s'arrêter à temps. Le cours de ce texte n'a plus qu'à avoir cours sous la terre. $)^{1}$

- Hélène Cixous, «Fichus et Caleçons ».

\section{Infinity pool: la piscine à débordement}

Je démarre au pas de course ${ }^{2}$ en posant la question du singulier-pluriel: dans l'expression "Derrida en cours", doit-on lire le ou les cours? Faut-il parler $d u$ ou des séminaire(s) de Derrida? Cette possibilité du pluriel qui interrompt le cours - "plus d'un cours", pourrait-on dire, qu'il s'agisse du cours du temps ou du cours tout court - ce pluriel, même virtuel, devrait compliquer toute tentative de réduire les séminaires de Derrida à l'unité d'une trajectoire, de les rassembler sous forme d'un cours linéaire.

Cependant, et malgré la profusion évidente des thèmes, notions et auteurs abordés par Derrida au cours des séminaires, il est tout de même possible d'observer d'un cours à l'autre, d'une année à l'autre, des effets de retour ou de hantise, certaines obsessions ou leitmotivs. Thèmes et variations: c'est d'ailleurs l'un des «thèmes » du séminaire, la nécessité d'une répétition ou d'une reproduction, inscrite à même la forme-cours ou la fonction-séminaire. C'est un certain plaisir, mais aussi (parfois) une lassitude du ressassement. Chaque année le séminaire se joue, se rejoue, fait retour et "performe» virtuellement l'anniversaire de tous les autres — à chaque fois, une fois pour toutes: le temps ritualisé du séminaire revient comme les saisons qui rythment la programmation d'un théâtre ou d'une

1. Hélène Cixous, «Fichus et caleçons», dans Cahier de L'Herne. Derrida, Marie-Louise Mallet et Ginette Michaud (dir.), L'Herne, 2004, p. 61. Cixous parle ici de «J. D.». C'est Cixous qui s'interrompt elle-même, déborde le cours de sa syntaxe, passe à la ligne et ouvre une parenthèse. Sur les courses de Derrida, voir Ginette Michaud, «Courir à toute vietesse: Note télégraphique sur un poème de pensée de J. D.», Mosaic: An Interdisciplinary Critical Journal, vol. 40, n 2, 2007.

2. Cet essai prolonge des travaux présentés lors de deux conférences organisées par Francesco Vitale à Salerno - l'une en juillet 2016 ( «The Future of Deconstruction. On Derrida's Archives»), l'autre en juin 2019 ( "From the archive. Reading Derrida»). Je tiens à remercier Francesco pour son hospitalité, ses conseils et son amitié, et pour avoir rendu possibles les discussions qui ont suivi — avec Peggy Kamuf, Geoffrey Bennington, Mauro Senatore, Ronald Mendoza-de Jesús, Raoul Frauenfelder, Silvano Facioni, Katie Chenoweth, Adam Rosenthal, Rodrigo Therezo et Jonathan Basile. Je tiens aussi à remercier Pierre Alferi pour m'avoir autorisé à reproduire des passages des séminaires inédits. Merci enfin à Ginette Michaud, Nicholas Cotton et Maxime Plante de m'avoir permis de relancer cette course, à l'occasion du collectif «Derrida en cours». 
salle de concert ${ }^{3}$. Il serait d'ailleurs vain de tenter d'ignorer le caractère immanquablement routinier du métier d'enseignant. Non seulement vain, mais ce serait probablement en manquer quelque chose d'essentiel: la course du soleil, sa révolution, la circularité de l'anneau ou du cercle, motif philosophique puissant sur lequel nous reviendrons.

Comment donc penser la continuité du (ou des) cours de Derrida tout en respectant la singularité de chaque séminaire, voire de chaque séance, chaque scène ou événement pédagogique? L'enseignement professé par Derrida peut parfois ressembler à un voyage au long cours — « heureux qui comme Ulysse", semble presque dire Cixous dans la citation placée en exergue de ce texte - mais il s'agirait d'un voyage sans retour définitif, traversé de trouées ou d'échappées débordant la tranquille linéarité d'un courant: dissémination du séminaire, par jeux de réponses et de correspondances, interruptions ou débordements.

Je donnerai rapidement deux exemples de tels débordements spécifiques à la forme-cours, par-delà les secousses thématiques ou tonales qui rythment l'écriture de Derrida, et auxquelles ses lecteurs et lectrices peuvent se croire habituées. L'enseignant-Derrida, pris par le temps trop court, préfère parfois se référer "par souci d'économie" aux textes publiés par ailleurs par l'auteur-Derrida - ce qui implique, au moins, la mise en scène performative d'une confiance accordée à l'auditrice ou à l'auditeur: «elle ou il m'aura peut-être lu». Non seulement le séminaire renvoie parfois à des publications existantes, mais il est lui-même une scène d'écriture, et certains extraits en seront par la suite remaniés et publiés. Ce jeu de correspondances suggère donc une certaine complémentarité entre les séminaires et les publications de Derrida - une complémentarité relative que nous pouvons tenter de reconstituer, nous lecteurs et lectrices, à contretemps, et à partir de l'archive. C'est un premier exemple de débordement, déjà difficile à maîtriser. Mais parfois le séminaire se répond aussi à lui-même, s'interrompt et déborde son propre cours: Derrida coupe court, brise le flot de son enseignement, et renvoie à d'autres années, d'autres séminaires, d'autres cours, souvent implicitement et parfois très explicitement $t^{4}$. Difficile de savoir, dans

3. Il faudrait analyser la façon dont Derrida évoque, dans virtuellement tous ses séminaires, le passage des saisons et les fêtes qui rythment le calendrier - Yom Kippour, la Toussaint, Noël et le Nouvel An, Pâques, le Ramadan, etc. Ces effets de revenance, quand ils sont re-marqués par Derrida, ont une grande puissance significatrice - ne serait-ce que parce qu'ils inscrivent au corps du cours les tours et retours des années, remarquant son inscription dans le texte général du calendrier, une routine si puissante qu'on semble en avoir oublié le sens (religieux, éthico-politique, historique, institutionnel, etc.).

4. Parmi les nombreux exemples d'auto-interruption explicite, en voici un, issu du séminaire «La vie la mort» (1975-1976), que je choisis d'évoquer parce qu'il concerne plus particulièrement notre objet d'étude. Lors de la cinquième séance, au cours d'une courte lecture de Marx, Derrida renvoie à deux reprises au séminaire de «l'année dernière » : «La re-productibilité n'est pas, là non plus, un accident survenant à la production mais l'essence même de la production. Ceci est extrêmement clair aussi dans les textes du Capital que nous avions lus ensemble 
de tels cas, si les séminaristes auront pu ou non assister aux cours des années précédentes. Est-ce, ou aura-ce été, le même séminaire - le même fleuve? L'Un de soi-même différant ${ }^{5}$ ? Et comment être sûr que nous maîtrisons bien ces jeux de correspondances, d'un cours à l'autre, et que nous ne reconstruisons pas violemment la linéarité du cours en lieu et place de l'interruption - d'une auto-interruption dont les ressources restent toujours plus ou moins implicites et surdéterminées? Toujours, une interprétation vient en lieu et place de l'interruption. Une certaine violence interprétative intervient, par exemple, dès que nous lisons un séminaire comme un livre - risque inévitable de l'après-coup, dont nous devrions, autant que possible, rendre compte.

Bien sûr, ces efforts de reconstitution ou de reconstruction, de traduction et d'interprétation, rendent chaque fois possibles de multiples trahisons, et donnent lieu à d'autres débordements... Ça déborde ${ }^{6}$ : cela arrive dès qu'il y a lecture, c'est-à-dire aussi écoute et traduction - à commencer par la prise de notes en cours - et cela arrive au cours du séminaire, dont le cours est d'emblée divisé par une discontinuité traversant le corps même de l'enseignant. Le cours du cours suppose ainsi d'autres courants ou contrecourants, plus souterrains ceux-là. C'est la course, car on ne sait jamais où va un cours. J'y reviendrai en conclusion.

«Derrida en cours»: la tension que j'évoquais pour commencer entre singulier et pluriel, entre continuité et discontinuité, travaille puissamment et singulièrement les séminaires que Derrida donna à l'École Normale Supérieure en tant qu'agrégé-répétiteur, jusqu'en 1984. Chaque année, le "caïman" devait adapter son enseignement, au moins partiellement, aux exigences de l'agrégation, notamment en traitant telles ou telles notions ins-

l'année dernière à propos de l'idéologie. [...] Je laisse de côté la question de la re-production non simple, élargie, puisque nous en avons parlé l'année dernière, mais vous imaginez bien quel intérêt elle aurait ici pour nous, du point de vue de ce que nous disions des "suppléments" de programme de production génétique.» Voir Jacques Derrida, La vie la mort. Séminaire (19751976), Pascale-Anne Brault et Peggy Kamuf (dir.), Paris, Seuil, 2019, p. 142-143.

5. On pense bien sûr à Héraclite et à son «fleuve ", qui n'est jamais tout à fait le "même". Derrida commente cette différance de «l'Un comme l'autre» dans un texte sur l'archive, justement: "L'Un se garde de l'autre. II se protège contre l'autre, mais, dans le mouvement de cette jalouse violence, il comporte en lui-même, la gardant ainsi, l'altérité ou la différence à soi (la différence d'avec soi) qui le fait Un. "L'Un de soi-même différant". L'Un comme l'Autre.» Cela pourrait se dire de tout «Un", du/des cours, de «Derrida en cours ", voire de «Derrida " tout court. Voir Jacques Derrida, Mal d'archive. Une impression freudienne, Paris, Galilée, 1995, p. $124-125$.

6. Sur ce thème complexe du "débordement ", difficile à traduire, et qui intéresse fortement Derrida, voir le séminaire "Théorie et pratique», dont une version a été publiée récemment: Jacques Derrida, Théorie et pratique. Cours de l'ENS-Ulm 1975-1976 [sic], Alexander García Düttmann (dir.), Paris, Galilée, 2017 (plus particulièrement p. 121-125). Contrairement à ce que l'édition française affirme, le séminaire prit place en 1976-1977. Voir aussi mon article: Thomas Clément Mercier, «Re/pro/ductions: Ça déborde», Poetics Today, vol. 42, $\mathrm{n}^{\circ} 1,2021$. 
crites au programme du concours, et ce dans le cadre d'un séminaire à fortes contraintes institutionnelles, spatiales, temporelles, etc. Ces contraintes sont les bords dans lesquels l'enseignement de Derrida se coule. Elles sont une source de frustration constante pour Derrida, frustration qu'il exprime de multiples façons au cours des séminaires des années 1970, en particulier ceux des années qui précèdent les engagements du GREPH ${ }^{7}$. Par exemple, le séminaire "Religion et philosophie» (1972-1973) commence par une mise au point combattive, quatre pages entièrement rédigées, dans lesquelles Derrida dénonce vertement les conditions de l'enseignement et le système agrégatif en général, notamment ses tendances conservatrices et reproductives. Ici, cette dénonciation violente de l'institution philosophique et enseignante semble séparée - textuellement et thématiquement - du "corps» du séminaire à proprement parler. L'introduction du séminaire de 19731974, "L'art (Kant) », fonctionne de manière similaire, mais ici les dénonciations sont davantage articulées au thème du séminaire. Derrida y annonce un certain nombre des préoccupations de ce qui deviendra plus tard le GREPH, et prépare les protocoles du séminaire de l'année suivante. En effet, la critique déconstructive de la forme-séminaire et de l'institution philosophique-enseignante passe au centre de la scène et devient un motif essentiel du séminaire de 1974-1975, "GREPH. Le concept de l'idéologie chez les idéologues français ${ }^{8}$ ", dont l'analyse constituera le cœur du présent essai.

7. Le GREPH (Groupe de recherches sur l'enseignement philosophique) s'est constitué lors d'une première assemblée générale en janvier 1975, mais cette constitution avait été précédée de réunions préparatoires en 1974. Derrida en fut l'un des principaux animateurs. Le GREPH avait pour objectif d'interroger «les liens qui existent entre la philosophie et son enseignement", et ainsi de réinscrire celui-ci dans "les conditions et les fonctions historiques et politiques du système d'enseignement en général ». L'une des visées "phares » du GREPH était d'étendre l'enseignement de la philosophie (jusque-là réservé à la terminale) à toutes les classes du lycée, voire d'en faire bénéficier des élèves plus jeunes. À partir de 1975, les initiatives du GREPH se sont cristallisées autour de la contestation de ladite "réforme Haby ", ce qui a contribué à donner une plus grande visibilité à ses travaux. Voir Jacques Derrida, Du droit à la philosophie, Paris, Galilée, 1990, p. 152. Voir aussi Vivienne Orchard, Jacques Derrida and the Institution of French Philosophy, New York, Modern Humanities Research Association and Taylor \& Francis, 2011.

8. Jacques Derrida, «GREPH. Le concept de l'idéologie chez les idéologues français» (1974-1975), Notes de séminaire, IMEC, Fonds Derrida (219 DRR 225.4), désormais abrégé "GREPH ». En 1974-1975, la notion au programme de l'agrégation de philosophie était «La société ». Notons que dans les archives de l'Université d'Irvine, en Californie, le séminaire est classé sous le titre "GREPH (le concept de l'idéologie chez les idéologues français) ». Dans le fonds Derrida situé à l'IMEC, il est désigné sous le titre «GREPH, le concept de l'idéologie chez les idéologues français ». Néanmoins, il faut noter que les copies du tapuscrit qui y sont disponibles portent seulement la mention "GREPH» en tête de chaque séance, suivie du numéro de séance. Le "sous-titre" n'est pas indiqué sur les notes qui composent le cours. Dans l'attente d'informations complémentaires, j'ai choisi de suivre l'usage de séparer «titre » et "sous-titre » par un point. Pour plus de détails sur ces difficultés terminologiques, voir Thomas Clément 
Des dénonciations similaires essaiment les séminaires des années suivantes, notamment «La vie la mort» $(1975-1976)^{9}$ ou «Théorie et pratique» $(1976-1977)^{10}$ — mais on en trouve aussi de nombreux exemples, peut-être moins massifs, dans les années qui suivent. En fait, il semble que ce questionnement du séminaire dans le séminaire, du cours dans le cours, soit devenu, plus particulièrement à partir des années 1970, un attribut quasi-essentiel de "Derrida en cours » — mais ce de manière toujours spécifique, en lien avec les questions au programme du séminaire. Ainsi, malgré la variété des thèmes, des notions et des auteurs abordés - variété partiellement imposée par les variations du programme (celui de l'agrégation) une basse continue semble traverser le ou les cours. Ça file, d'une année à l'autre: les séminaires se succèdent et reproduisent la mise en question du cours conçu comme organe inséminateur ou appareil de reproduction. Derrida y poursuit une analyse militante des protocoles de l'enseignement, à travers la déconstruction de l'institution philosophique en tant qu'instance propédeutique et machine reproductrice. Ce qui semble se reproduire, c'est donc une déconstruction du séminaire, de sa fonction inséminatrice de reproduction.

C'est sans doute dans le séminaire de 1974-1975, "GREPH. Le concept de l'idéologie chez les idéologues français ", que l'analyse réflexive de Derrida sur son propre cours, sur les conditions de son enseignement, est la plus évidente et explicite. Dès la première page du séminaire, Derrida écrit:

Après environ 15 ans de pratique enseignante et 23 ans de fonctionnariat, je commence seulement à interroger, exhiber, critiquer systématiquement (c'est le

Mercier, «Différence sexuelle, différence idéologique: Lectures à contretemps (Derrida lisant Marx et Althusser, dans les années 1970 et au-delà) ", Décalages, vol. 2, n 3, 2020, p. 18-19.

9. Dans ce séminaire, Derrida propose une analyse déconstructrice des notions de "programme ", de "reproduction" et de "sélection ", aussi bien dans le champ de la biologie et de la génétique que dans celui des institutions sociopolitiques, en particulier l'enseignement. Voir notamment les séances 4 à 6 du séminaire, consacrées plus particulièrement au concept de «reproduction» dans le discours scientifique du biologiste François Jacob. Derrida y propose une analyse déconstructrice incessante et implacable de la logique de la productionreproduction, à laquelle j'ai consacré de récents travaux. La cinquième séance, intitulée "L'increvable", s'ouvre sur la déclaration suivante: "Le concept de re-production est à peine concevable» (La vie la mort, p. 133). Je ne peux malheureusement pas y insister ici, mais il est important de garder à l'esprit que Derrida, dans ce séminaire, tient à ne jamais séparer la question de la reproduction biologique du vivant (reproduction dite «sexuelle" ou non), de celle de la reproduction institutionnelle ou socio-économique (notamment la reproduction permise ou promise par l'appareil éducatif). L'une est la métaphore de l'autre. Entre ces deux «reproductions ", l'onto-logique de la re-production comme essence du discours philosophique. Je renvoie à ce sujet à mon article "Re/pro/ductions: Ça déborde».

10. Voir à ce sujet: Michael Naas, "Education in Theory and Practice: Derrida's Enseignement Supérieur », Studies in Philosophy and Education, 2020, DOI: https://doi.org/10.1007/ s11217-020-09723-y 
caractère systématique qui importe si l'on veut ne pas se contenter de coups de griffe empiriques qui n'ébranlent ni ne transforment le système en place, font au contraire partie de sa reproduction auto-critique, la reproduction autocritique étant peut-être l'élément même de la tradition et de la conservation philosophique, l'art de la question dont nous dirons un mot plus loin), je commence donc seulement, si tard, à interroger, exhiber, critiquer systématiquement et en vue de transformation, les bords même de ce dans quoi je coulais mon discours jusqu'ici ${ }^{11}$.

Dé-bordements. Après avoir, dans cette introduction, multiplié les précautions et exposé les risques qu'il y aurait à isoler tel ou tel séminaire dans le cours général du cours, je m’apprête donc à ignorer ces difficultés pour me concentrer sur le seul séminaire "GREPH. Le concept de l'idéologie chez les idéologues français ${ }^{12}$ ». Au cours de cette étude, je suivrai notamment trois pistes, trois fils : 1) l'analyse déconstructrice de l'enseignement, et en particulier de l'institution philosophique enseignante dans sa fonction reproductrice; 2) le rapport que cette institution entretient avec la "reproduction" du corps sociopolitique, telle que décrite par les discours critiques marxistes et/ou sociologiques - l'analyse de ce rapport passera par une déconstruction du concept d'idéologie, notamment chez Marx et Althusser; et 3) la «question" de la différence sexuelle, à laquelle Derrida se réfère pour déconstruire le concept traditionnel de reproduction. J'essaierai d'expliquer la nécessité de l'entrelacement de ces trois fils, et donc celle d'une triple déconstruction, du cours au cours. Comme nous le verrons, ces trois fils se nouent au lieu de la lecture, c'est-à-dire du texte. À travers le texte, Derrida s'efforce de penser une autre économie, non reproductrice, excédant la logique de la reproduction, qu'il identifie aussi bien chez les économistes classiques que chez leurs critiques marxistes.

\section{Scènes: du séminaire (les corps divisés)}

Le séminaire de 1974-1975 est l'occasion pour Derrida d'aborder des questions philosophiques qui ressortent de ce que l'on nomme conventionnellement la politique, la société et l'économie. C'est aussi l'occasion pour lui d'analyser des textes appartenant à la tradition marxiste: Marx et Engels, mais aussi Gramsci, Althusser, Balibar, ou Buci-Glucksmann. Toutefois, Derrida semble le faire de manière quelque peu oblique. Le séminaire est un texte complexe, extrêmement riche, qui tresse de nombreux fils théoriques avec pourtant une cohérence impressionnante. Le premier de ces fils est lié à

11. «GREPH», séance 1, p. 1. Je souligne.

12. Le séminaire reste aujourd'hui inédit dans son immense majorité. Une partie des séances 1 et 2 fut remaniée et publiée sous le titre "Où commence et comment finit un corps enseignant». Ce texte parut d'abord dans Dominique Grisoni (dir.), Politiques de la philosophie, Grasset, Paris, 1976. Il fut ensuite republié dans Du droit à la philosophie. Je m'y réfèrerai çà et là. 
la situation d'énonciation: le séminaire fut présenté dans le contexte du GREPH. Il est donc situé et engagé, politiquement et institutionnellement. Du point de vue thématique, Derrida s'intéresse aux conditions de l'enseignement de la philosophie. Il s'agit d'offrir une analyse socio-historique des rapports de force théoriques et pratiques qui sous-tendent les institutions scolaires et universitaires en France (et en Europe) depuis la Révolution française. Au cours de cette analyse, Derrida souligne les luttes agonistiques et les forces répressives qui ont conduit à l'établissement d'un programme d'enseignement spécifique: conditions institutionnelles de l'enseignement, pratiques pédagogiques et protocoles de l'examen, construction d'un corpus philosophique dit "canonique» (souvent indexé sur les auteurs dits «nationaux»), exclusion de certains auteurs ou de certaines écoles de pensée (la scolastique trop marquée théologiquement, trop «cléricale», ou le matérialisme trop marqué «à gauche»), expulsion de certains individus dudit «corps enseignant " (à commencer par les femmes, étudiantes et enseignantes, jusqu'à très tard), jeux d'influence des acteurs politiques, religieux et économiques sur les institutions d'enseignement, etc.

Derrida précise que ces exigences souvent contradictoires travaillent au corps ce matériau socio-historique et constituent un champ de forces différentiel et instable, dont les limites objectives restent difficiles à tracer. En effet, ce qu'on appelle "l'enseignement» est lui-même un champ de luttes, et ces luttes ont notamment pour objet la définition des limites du «champ» en question. Au cours de son histoire, ledit "corps enseignant» s'est vu reproduire à travers des processus agrégatifs de survie et d'autoperpétuation, mais aussi des phénomènes d'exclusion et d'expulsion, autant de mutations plus ou moins forcées, violentes ou convulsives. Le corps se défend en tant que corps, produit des anticorps - mais, ce faisant, il se $f e n d^{13}$. Le corps enseignant s'en trouve intrinsèquement divisé, hanté par ses doubles, ses corps autres, eux-mêmes complexes, multiples et intérieurement divisés: non seulement le corpus philosophique dont l'enseignement est censé être le garant et le véhicule, mais aussi le corps socio-politique qu'il est censé légitimer et «reproduire» dans ses fonctions - à travers par exemple l'enseignement des aptitudes aux jeunes gens (en priorité des hommes ou futurs hommes) selon l'organisation générale de la société, la sélection des individus et la répartition des forces productives, la définition des «disciplines» faisant écho à telle ou telle définition de la division du travail, la reproduction légitimatrice d'une société de corps, etc.

Tout cela rend difficile la détermination ou la définition du «corps» ou $\mathrm{du}$ «champ » en question, et des limites de ce qui «se reproduit» à travers lui:

13. Ce processus quasi-auto-immunitaire est décrit aux premières pages de la séance 3 du séminaire. 
Il y a donc, partout où l'enseignement a lieu — et dans le philosophique par excellence - des pouvoirs, représentant des forces en lutte, des forces dominantes ou dominées, des conflits et des contradictions (ce que j'appelle des effets de différance) à l'intérieur de ce champ. C'est pourquoi un travail comme celui que nous entreprenons [...] implique de la part de tous ceux qui y participent une prise de parti politique, quelle que soit la complexité des relais, des alliances et des détours stratégiques [...].

Il ne saurait donc y avoir un corps enseignant ou un corps d'enseignement (enseignant/enseigné : nous élargirons la syntaxe de ce mot, du corpus enseigné au corps des disciples): homogène, identique à soi, suspendant en lui les oppositions qui auraient lieu au-dehors (par exemple les politiques), et défendant à l'occasion LA PHILOSOPHIE EN GÉNÉRAL contre l'agression du nonphilosophique en provenance de l'extérieur. S'il y a donc une lutte quant à la philosophie, elle ne peut manquer d'avoir son lieu à l'intérieur comme à l'extérieur de l' «institution" philosophique. Et s'il y avait quelque chose de menacé à défendre, cela aussi aurait lieu dedans et dehors, les forces du dehors ayant toujours leurs alliés ou leurs représentants au-dedans. Et réciproquement ${ }^{14}$.

Au cours du séminaire, Derrida décrit, parmi lesdites "forces du dehors » (qui se trouvent donc déjà «dedans»), divers pouvoirs politiques, religieux, ou socio-économiques. Il s'agit donc aussi d'offrir une analyse historique des États-nations européens ${ }^{15}$, de la philosophie des nations et de l'Étatnation comme "thème" ou "thèse " de la philosophie, autour de concepts philosophico-institutionnels tels que «le lycée» ou "l'université », pensés comme fabriques du citoyen national et universel. Ainsi, Derrida ne souhaite pas séparer la question de «la » philosophie (et de son enseignement — l'enseignement et l'université comme "thèmes » philosophiques) de celle de l'organisation socio-économique générale. Ces deux questions sont inséparables. Des notions telles que «État» et «société » sont des philosophèmes, des objets philosophiques qu'il faut aussi analyser comme tels. Et il y va toujours d'un certain corps, un corps qui se représente (ou se représente) comme tel à travers l'enseignement et la philosophie ${ }^{16}$. En dessinant les contours de cette micro- et macro-physique enseignante, Derrida propose ainsi une réflexion d'économie philosophique générale, une éco-

14. Derrida, Du droit à la philosophie, p. 128.

15. Cela passe notamment par une lecture de la correspondance entre Victor Cousin et son «maître» Hegel, que Derrida commente dans la perspective d'une analyse des relations franco-allemandes au sujet de l'université.

16. C'est pourquoi l'enseignement constitue une entrée privilégiée pour la pratique déconstructive: "la déconstruction du phallogocentrisme comme déconstruction du principe onto-théologique, de la métaphysique, de la question "qu'est-ce que ?", de la subordination de tous les champs de questionnement à l'instance onto-encyclopédique, etc., une telle déconstruction s'attaque à la racine de l'universitas: à la racine de la philosophie comme enseignement, à l'unité ultime du philosophique, de la discipline philosophique ou de l'université philosophique comme assise de toute université. L'université, c'est la philosophie, une université est toujours la construction d'une philosophie» (Derrida, Du droit à la philosophie, p. 119). 
nomie politique de la philosophie et de son enseignement. Il s'agit d'analyser le rôle et la fonction du discours philosophique qui, en tant qu'idéologème, en tant que production idéologique, produit aussi des effets pratiques dans l'organisation socio-politico-économique — bref, matérielle - dans laquelle il s'inscrit et dont il est aussi le reflet, à la fois fidèle et infidèle.

Conformément à certaines prémisses de l'analyse marxiste, Derrida étudie donc le devenir du discours philosophique tel qu'il est décrit par Marx et Engels comme exemple parmi d'autres de "reflet", "forme» ou "expression" idéologique (à côté de la religion, de la science politique et juridique, de l'esthétique, etc. ${ }^{17}$. Toutefois, le séminaire de Derrida effectue un déplacement du lieu de l'analyse marxiste entendue comme simple critique de l'objet-idéologie: en s'intéressant non seulement à des textes philosophiques classiques (et en particulier à ceux de l'idéalisme étatiste, ceux de Hegel ou les traités de pédagogie de Victor Cousin), mais aussi aux conditions pratiques de l'enseignement liées à son histoire politico-institutionnelle, Derrida s'efforce de ré-institutionnaliser le discours philosophique et d'analyser les pratiques discursives, matérielles et empiriques à travers lesquelles celui-ci s'inscrit et se reproduit. Derrida tente donc de penser le philosophique dans son effectivité pratique et matérielle, comme Wirklichkeit et non seulement comme forme idéologique, «reflet» ou "expression » d'une réalité matérielle préexistante. Cela suppose un certain déplacement des limites conventionnelles du champ de l'idéologique tel que la tradition marxiste l'aura circonscrit par opposition à d'autres domaines - par exemple l'«appareil répressif» ou l'«infrastructure économique» conçue comme «dernière instance ${ }^{18}$.

$\mathrm{Au}$ contraire, Derrida souligne une immixtion réciproque, une coimplication structurelle, intime et irréductible, entre l'organisation socioéconomique et ce qui se nomme «la philosophie». En analysant les textes de philosophes-économistes - notamment Condillac, Adam Smith, Destutt de Tracy, Jean-Baptiste Say, Ricardo et bien sûr Marx -, Derrida démontre que, chez tous ces auteurs, la place et le rôle donnés à l'enseignement (et en particulier à l'enseignement de la philosophie) sont toujours liés à une certaine organisation socio-économique, et supposent ainsi un certain positionnement de l'économiste-philosophe quant à la division du travail, la hiérarchisation sociale, l'économie politique, et quant au «corps» de la

17. Voir Karl Marx et Friedrich Engels, L'idéologie allemande, Paris, Éditions sociales, 1968, notamment p. 50-52. Toutes ces notions sont aussi analysées par Derrida dans les deux premières séances du séminaire inédit «Religion et philosophie» (1972-1973). Voir à ce sujet Mercier, «Différence sexuelle, différence idéologique », p. 8-16.

18. Louis Althusser reprend ces notions dans son fameux article «Idéologie et appareils idéologiques d'État (Notes pour une recherche)» - essai d'abord publié dans La Pensée en 1970, et republié notamment dans Louis Althusser, Sur la reproduction, Paris, Presses Universitaires de France, 1995. Derrida consacre la quasi-intégralité de la séance 5 du séminaire à l'analyse de cet essai d'Althusser. J'y reviens ci-dessous. 
communauté étatique et nationale. Et puisqu'on parle de «corps ", Derrida souligne également que tous ces philosophes-économistes définissent aussi une certaine distribution de la différence sexuelle - soit parce qu'ils adressent leur discours aux seuls hommes ou «jeunes gens ", soit à travers une définition explicite de la famille, de la sexualité comme reproduction, de la répartition des rôles entre l'homme et la femme et de leur fonction dans la reproduction du capital, etc. ${ }^{19}$ Selon Derrida, ces prises de position sociopolitico-économico-sexuelles ne sont pas simplement des caractéristiques accidentelles de «la philosophie» en tant que forme ou expression idéologique: elles sont en fait inséparables du discours philosophique en son économie «propre» - comme économie du propre, justement, du «corps propre » et de sa «reproduction» - et donc de sa fonction prétendument organisatrice du socius, du politique, et de l'économie «en général ». Ainsi, l'institution philosophique (et sa puissance légitimatrice, qui s'affirme notamment à travers l'enseignement, partout avec la violence d'une inculcation) accompagne l'organisation "économique» au sens traditionnel du terme - non seulement parce qu'elle lui procure force et légitimité, mais parce qu'il s'agit bien ici de la «même » économie et du «même " corps, qui vise à se présenter et à se re-présenter "comme tel », comme corps homogène et organisé, par et dans le jeu de l'économie du propre - et ce malgré ses divisions et son hétérogénéité structurelles. Cette logique de la présence et du propre, qui reste à déconstruire, est reconduite aussi bien par le philosophe que par l'économiste (libéral ou critique-marxiste), qui partagent les mêmes concepts, les mêmes mots, le même langage ${ }^{20}$.

Dans ce séminaire, Derrida fait donc du même coup (au moins) deux gestes à la fois: d'une part, il réinscrit le discours philosophique dans son élément apparemment extra-philosophique, dans son histoire institutionnelle, les rapports de forces qui la sous-tendent, à travers une analyse détaillée des conflits qui traversent ledit «corps enseignant» et l'enseignement de la philosophie depuis la Révolution française. Ces conflits ont des implications politiques (ou politiciennes), sociologiques ou économiques, religieuses ou théologiques, mais aussi individuelles ou interpersonnelles, nationales et coloniales, familiales et sexuelles, etc. Ils supposent toujours une lutte pour une certaine hégémonie, l'esquisse d'un impérialisme. Mais, d'autre part, Derrida fournit une analyse déconstructrice des outils et concepts philosophiques qui permettent aujourd'hui une telle analyse historique ou historicisante du "champ» de la philosophie, de ses discours et de son enseignement. Ainsi, le séminaire est aussi l'occasion pour Derrida d'interroger le lieu d'inscription du discours marxiste dans le champ et l'histoire

19. Dans la séance 4 du séminaire, Derrida évoque ce qu'il appelle «l'organisation idéologico-sexuelle» du socius.

20. Ces questions liées à l'épistémologie des sciences sociales et économiques sont reprises de manière plus développée dans Mercier, «Différence sexuelle, différence idéologique », p. 25-26. 
de la philosophie, et ainsi d'offrir une généalogie déconstructrice du concept d'idéologie. Derrida montre que la critique marxiste de la philosophie (sous forme d'une critique de l'idéologie) n'a pu se penser et être conduite qu'en empruntant ses propres concepts à la tradition philosophique - notamment, le concept d'idéologie, qui a été "produit » par Destutt de Tracy et les «idéologues» français à la suite de Condillac, au cours d'une histoire dont on peut faire la généalogie, et qui devrait aussi inclure selon Derrida une certaine lecture déconstructrice de Hegel. Cette histoire est aussi celle de "l'appareil idéologique », qui n'aura pu être produit et identifié comme tel, comme objet de la critique marxiste, que grâce aux instruments qu'il aura lui-même fournis à la critique ${ }^{21}$. Ainsi, le discours le plus révolutionnaire court toujours le risque de reproduire les schèmes métaphysiques qu'il vise pourtant à renverser. La déconstruction du discours philosophique doit donc aussi opérer depuis l'intérieur de la philosophie, même si cela vient du même coup compliquer la frontière intérieur/extérieur. Il s'agirait d'une enclave ou d'une dissidence interne: une crypte.

Expliquons. Au seuil de la séance 5 du séminaire - séance consacrée au texte "Idéologie et appareils idéologiques d'État», d'Althusser —, Derrida explique cette difficulté par le fait que l'idéologie œuvre en préservant le secret de son énonciation. L'idéologique réside en ce secret. Nous restons structurellement aveugles, du moins partiellement, mais irréductiblement, aux conditions idéologiques depuis lesquelles nous parlons. C'est pourquoi il n'y a pas l'idéologique «comme tel » : l'histoire de l'idéologie ne peut être produite qu'à travers une certaine facture idéologique (incluant un certain concept d'histoire) dont nous restons partiellement captifs, et qui suppose des effets d'occultation intrinsèques, irréductibles. L'occultation fait partie de son histoire; effet de structure, l'occultation est l'idéologique comme histoire, et se voit produire ou reproduire par son existence même comme histoire (et non simplement dans l'histoire). Cela, dit Derrida, n'est pas seulement dû à un manque ou à une dissimulation d'archives et de matériaux historiques - lettres, documents, objets perdus, détruits ou cachés - qui nous permettraient, si nous les retrouvions, de saisir la clef ou de décrypter le code de tel ou tel appareil idéologique, des structures qu'il suppose et qui le soutiennent. Non, car au-delà de ces manques circonstanciels, l'occultation idéologique est structurelle. Puisque ces belles analyses de

21. C'est cette histoire en partage qui permet qu' «aujourd'hui » une critique de l'appareil soit formulée, dans un langage que la critique doit partiellement partager avec lui, emprunter à cela même qu'elle critique. Cet "aujourd'hui » suppose donc une complicité entre sujet et objet de l'analyse critique, mais aussi un retard, une différance, car sujet et objet ne se font pas face comme deux présences extérieures l'une à l'autre. Leur contamination différantielle suppose aussi une non-contemporanéité, un contretemps. C'est cela qui à la fois permet et compromet l'objectivité — c'est-à-dire la constitution dudit appareil idéologique comme objet disponible à l'examen scientifique ou théorique. 
Derrida restent aujourd'hui inédites, je citerai longuement son introduction à la lecture d'Althusser, aux premières pages de la séance 5 du séminaire:

Le recours à l'archive ou la reconstitution d'une archive dissimulée n'est jamais due à l'initiative d'un sujet, d'un chercheur, d'un historien ou d'un rat de bibliothèque: il faut qu'une situation historique et structurale générale rende nécessaire et possible, dans des conditions analysables en principe, qui s'ouvrent alors à l'analyse, la constitution de l'archive en objet. Mais - donc - il ne s'agit pas alors d'une dissimulation d'archive, de l'occultation de quelque chose, d'un objet, d'un ensemble d'objets (événements, écrits, etc.) que dans une situation donnée on pourrait enfin découvrir comme contenu déjà là. La dissimulation n'est pas ici une simple dissimulation, la cachette d'un objet, c'est l'absence ou la non-disponibilité des moyens, des instruments théoriques nécessaires pour reconstituer et interpréter l'archive. Autrement dit, l'objet en question, si on peut encore parler d'objet, s'est construit de telle sorte que non seulement il s'occulte ou se dérobe mais qu'il fait obstacle à la disposition des moyens nécessaires à le chercher et à le trouver. Si vous voulez une image: non seulement nous n'avons pas ici affaire à un objet que quelqu'un aurait caché dans un coffre-fort ou dans une bibliothèque, mais à un objet qu'un système a constitué de telle sorte qu'il ne commence à exister que dans un coffre-fort et de telle sorte que les clés du coffre-fort n'existent pas en dehors du coffre-fort et, à vrai dire y soient même enfermées ${ }^{22}$.

Il ne s'agit donc pas simplement de "déterrer ", de révéler des vérités préexistantes (vérités qui auraient été rendues inaccessibles en raison d'une telle occultation idéologique), mais plutôt d'inventer, d' «élaborer les instruments "théoriques" » qui nous permettent de constituer l'objet même de notre analyse, chaque fois de manière unique et singulière. Cette nécessité est due au fait que,

en tant que produits - pour toute une part — du système que nous étudions, nous avons été - pour cette part — privés par le système des moyens théoriques et pratiques de le déconstruire. Et si nous ne le sommes pas totalement aujourd'hui, c'est dans la mesure où le système en question est lui-même une construction qui se déconstruit, et qu'aujourd'hui sa déconstruction historique atteint une phase où notre projet est explicitement possible, explicitable ${ }^{23}$.

Ici se pose donc la question des possibilités de la lecture — c'est-à-dire aussi celle des instruments interprétatifs qui nous permettraient de lire, d'analyser ou de déchiffrer la crypte ou le secret, secret de l'archive ou de l'idéologie. Mais - et c'est notamment ce que Derrida trouve réducteur dans la «logique» de l'idéologique - ici nous ne parlons pas simplement de tel ou tel secret, du secret d'une vérité à dévoiler, délimitable, appropriable, circonscriptible par et dans une analyse finie, finissable; il y va aussi d'un certain secret originaire et constitutif de la chose «archive» ou «idéologie».

22. «GREPH», séance 5, p. 2.

23. Ibid., séance 5, p. 2-3. 
Secret il y a - et c'est là que la lecture déconstructrice commence: c'est même la condition de toute lecture.

Lecture à la fois interne et externe à la philosophie, donc. Ce qui est particulièrement intéressant, ici, c'est que cette idée est justement mise en abyme à travers la situation d'énonciation du cours: Derrida en cours tente de déconstruire les conditions de l'enseignement philosophique au moment même où il produit un enseignement philosophique, normé, normalisant ou normant, au cœur de l'École normale supérieure. Il s'agit donc de jouer dans la norme, contre la norme, depuis la norme, à partir d'elle. À corps divisé. Ce qui pourrait être qualifié de contradiction performative est pleinement reconnu et assumé par Derrida: le champ institutionnel de l'enseignement suppose "une multiplicité d'antagonismes toujours surdéterminés », de telle sorte que «la courroie de transmissions travaille et traverse toute sorte de résistances, de contre-forces, de mouvements de dérive ou de contrebande ». Enseigner n'est donc jamais un geste simple. Il implique une forme de "dissociation", une "scission" qui consiste à appliquer des règles, à enseigner ce à quoi on ne croit pas tout à fait - cela même qu'on critique par ailleurs. La dimension performative de l'enseignement, ici celui de ladite "philosophie », est donc hantée par sa propre mise en scène. Le "répétiteur met son tablier », dit Derrida, à travers une véritable «fiction dissociatrice $»^{24}$.

Par rapport à «l'institution", donc, qu'il s'agisse de l'École normale supérieure, du «corps enseignant », de «la philosophie» ou de «l'appareil» en général, Derrida tente ainsi d'être à la fois dedans et dehors, dedansdehors: à l'intérieur mais au-delà. Durant les années 1960 et 1970, son collègue et ami Althusser aura tenté quelque chose de similaire dans son séminaire à l'ENS. La critique des appareils idéologiques d'État (AIE), y compris l'AIE-École et l'enseignement, occupe en effet une place importante dans le discours critique althussérien ${ }^{25}$. Cependant, à la différence de Derrida, Althusser ne théorise ni ne thématise, justement, la logique de ce dedans-dehors, cette enclave dissidente à l'intérieur d'une institution dont la critique reste partiellement, mais irréductiblement, dépendante ${ }^{26}$. Ce que Derrida tente de montrer dans son séminaire sur «Le concept de l'idéo-

24. Ibid., séance 1, p. 6. Voir aussi Du droit à la philosophie, p. 123-125.

25. Derrida souligne à plusieurs reprises l'apport incontestable, au regard de la tradition marxiste, que constitue la théorie de l'idéologie et des AIE d'Althusser (que Derrida associe souvent à Gramsci à cet égard), et en particulier son analyse de l'AIE-École: «Qu'on se satisfasse ou non de ce qui est fait à cet égard par Gramsci et par Althusser, il me paraît nécessaire de tenir compte ici d'un frayage. Ne serait-ce que pour expliquer qu'en son absence les lectures marxistes des phénomènes qui nous intéressent ici — je pense en particulier à celles de Lucien Sève, quel que soit l'intérêt du matériau qu'il exhibe - soient restées très insuffisantes, mécanistes, empiristes, économistes. " "GREPH ", séance 4, p. 16.

26. Par contraste, Althusser évoque l'héroïsme individuel des rares «maittres ", ces « héros », qui s'efforcent de critiquer l'idéologie dominante dans leur classe. Voir «Idéologie et appareils idéologiques d'État", p. 292. 
logie", donc, c'est que toute critique institutionnelle - par exemple la critique de l'idéologie - est aussi un effet de l'institution. La critique de l'idéologie ne peut être qu'un effet de l'idéologie. La critique appartient à l'appareil, à supposer que ledit "appareil» ait une quelconque unité ou homogénéité. C'est ce qui constitue la chance de la lecture, mais aussi sa limite. Une limite interne. Cette idée est au centre de l'analyse que propose Derrida de la critique althussérienne de l'idéologie:

Les concepts marxistes de production, d'idéologie, etc., même s'ils sont construits en rupture, en contradiction ou en déplacement par rapport à Hegel, comportent ce rapport à Hegel, au concept d'idéologie qui date de cette époque et se construit dans un certain rapport et aux philosophèmes et aux appareils d'enseignement dont ils sont inséparables. Ils sont énoncés, mis en place par des intellectuels, voire des professeurs de philosophie, dans un langage et une culture qui gardent nécessairement un lien avec ce qu'ils constituent comme leur objet, par exemple les Appareils Idéologiques d'État, et ce lien, quel qu'il soit, même s'il n'est pas simplement celui d'un produit à sa source productrice, ou d'une simple reproduction à l'appareil de production ou de reproduction, ce lien ouvre certes d'un côté la possibilité de lecture et d'analyse de l'appareil mais la limite aussi, [faisant] que par certains côtés la théorie de l'appareil, quant à l'appareil, est aussi pour une part un effet de l'appareil ${ }^{27}$.

On ne peut donc pas «sortir» de l'appareil idéologique, si «sortir» veut dire échapper à l'ancrage historico-institutionnel qui est le nôtre pour produire un discours critique, théorique ou scientifique, tendanciellement transcendant et souverain, sur les conditions qui nous ont permis de formuler un tel discours. Ici encore, Derrida se réfère à la notion de «lecture»: la lecture suppose un certain rapport à l'archive, une dépendance vis-à-vis des matériaux que nous lisons comme un texte, un texte dont nous dépendons, donc, et dont nous sommes aussi les effets en tant que lecteurs. Ce qui m'intéresse particulièrement ici, c'est que ce qui limite la lecture est aussi ce qui la permet - ce qui «ouvre» sa "possibilité ». Or la lecture n'est ni simplement "production» ni simplement «reproduction». Il s'agit d'autre chose. Cette insistance sur la lecture - et sur l'enseignement, le cours comme lecture implique donc aussi une prise de position par rapport au concept de « reproduction ", essentiel à l'analyse althussérienne:

Tout ce que nous disons ici sera aussi, pour une part, limité par notre appartenance, notre lieu de formation, etc., par le système très complexe et surdéterminé de ces effets. Cela ne veut pas dire que nous sommes dans un cercle, un cercle herméneutique ou spéculatif se re-produisant indéfiniment. Ce philosophème "cercle» a une place très déterminée dans la tradition philosophique utilisée, mise en œuvre ou investie par les Appareils que nous étudions. Une analyse de ces appareils doit éviter non seulement de reproduire ces appareils

27. «GREPH », séance 5, p. 4-5. 
mais de se contenter d'être une analyse théorique ou une prise de position à l'égard, quant à, au sujet de ces appareils, elle doit transformer l'appareil en tant qu'il fonctionne comme machine reproductrice ${ }^{28}$.

La déconstruction de la machine reproductrice suppose donc une transformation de l'institution philosophique conçue comme «idéologie » ou « appareil reproductif », ce qui suppose une remise en question du statut donné au texte philosophique - remise en question dont Derrida va tenter de « déchaîner» les traces dans les écrits de Marx.

\section{En bout de course (dissémination, dérive, déchaînement)}

Dans les deux dernières séances du séminaire, avant l'interruption finale, Derrida accélère fortement sa course et approche la notion d'idéologie telle qu'elle apparaît dans le texte de Marx. Derrida va alors insister sur les effets de déplacement produits par ce texte quant au statut et à l'unicité de ce qu'on appelle "philosophie». Ces déplacements affectent au premier chef l'école et l'institution philosophique comme programme - le cours et le discours enseignant:

Il est marqué ici [par Marx] que la dimension philosophique du discours n'est pas la bonne, l'arbitrale en dernière instance, et que c'est le statut même du texte philosophique qui se trouve, doit se trouver déplacé; que les questions même qu'on lui pose ne doivent pas être en dernière instance des questions de style ou de forme philosophique, au moins au sens courant dans les écoles. Et au fond, si le texte de Marx nous intéresse ici, ici même, c'est-à-dire au cours du travail que vous savez sur l'enseignement philosophique, c'est non seulement pour toutes les raisons de contenu que nous avons déjà repérées (rapport aux idéologues, au concept d'idéologie, etc.) mais je dirais surtout, quoi que de manière moins visible, en raison de son statut par rapport à l'institution philosophique, au type ou au style de discours qui peut s'y tenir ou s'y transmettre ou s'y reproduire. Il y a là un énorme réseau de questions que je ne fais que signaler. Pour l'instant je me contente d'attirer l'attention sur le statut de ce texte et sur le fait qu'il n'autorise peut-être pas, déroute au contraire et disqualifie d'avance certains types de questions philosophiques programmées par la philosophie et son enseignement ${ }^{29}$.

Dans ce sprint final, Derrida va donc proposer une lecture de Marx inédite, audacieuse, fulgurante, au pas de course. Or, si Derrida se permet ainsi une lecture déchaînée de Marx, c'est notamment parce qu'il souhaite formaliser ou thématiser ce « déchaînement » comme mode de lecture. En effet, Derrida choisit d'exposer et d'opposer deux lectures possibles du texte de Marx au sujet de l'idéologie: l'une, décrite comme « réductrice» et «conservatrice»;

28. Ibid., séance 5 , p. 5 .

29. "GREPH », séance 9, p. 7. 
l'autre, "déclôturante et productrice ${ }^{30}$ ", sur le mode du "déchaînement ${ }^{31}$. Toutefois, Derrida dit d'emblée que ces deux lectures, permises ou promises à partir du texte de Marx, sont à la fois incompatibles et pourtant possibles — possibles malgré, ou grâce à, leur inconciliabilité ou leur hétérogénéité essentielles. Et c'est précisément le fait que le texte de Marx reste un texte qui permet son ouverture à l'impossible événement de lectures contradictoires ou incompossibles.

La première lecture, «réductrice», consisterait à reconduire les schémas hégéliens qui sous-tendent le texte marxien, et ainsi à en reproduire les structures dialectiques et téléologiques (dont Derrida nous dit qu'on en trouve évidemment la trace dans le texte de Marx). La philosophie marxiste offrirait ainsi une "ontologie du travail»: «il s'agit d'une ontologie dialectique ${ }^{32}$. Si l'on suit ce cadre interprétatif, l'idéologie ne serait qu'un simple détour entre deux présences, moment dialectique entre deux moments sans aliénation. Selon cette lecture, que Derrida qualifie de "métaphysique», l'idéologie témoignerait de la division du travail dans un rapport d'identité: l'idéologie traduirait sans reste la division du travail, même si elle tend à en occulter les ressorts ou les effets. Dans cette perspective, la critique marxiste de l'idéologie serait donc en mesure de révéler le phénomène de la division du travail «comme tel», dissimulé derrière l'idéologie "comme telle» (ce qui suppose, selon Derrida, une conception métaphysique traditionnelle de la conscience, de l'imagination, et de l'idéologie comme fausse représentation). La critique marxiste permettrait à terme l'abolition de l'idéologique et de la domination de classe en abolissant (sur le mode de l'Aufhebung) la division du travail - à commencer par la division entre travail intellectuel (geistig) et travail matériel, qui renvoie selon Derrida aux mêmes schémas métaphysiques ou ontologiques.

\section{Ibid., séance 9, p. 9.}

31. Le mot «déchaînement» n'est pas choisi au hasard. Derrida joue autant sur ses virtualités politiques - disons, pour aller vite, ses connotations émancipatrices ou «spartakistes» - que sur tout ce qui en lui fait signe vers la force irruptive d'une folie débordante ou d'une rage spontanée, voire d'une soudaine décharge libidinale - et ce en fin de course du séminaire. Il y aurait beaucoup à dire sur ce qui justifie, pour Derrida, un tel « déchaînement ", à telle époque, sur telle scène, dans le contexte d'une lecture de Marx. La puissance (voire la jouissance) farouche de ce "déchaînement" vise aussi bien les chaînes (sémantiques, linguistiques, théoriques, institutionnelles, politiques, etc.) qui ont depuis longtemps cadenassé le discours marxiste, que des adversaires plus ou moins identifiables dans le champ que Derrida a analysé au cours du séminaire. Derrida dit en effet que la lecture réductrice, reproductrice, du texte de Marx imprègne "toute l'histoire du marxisme " et a «des conséquences lourdes dans les stratégies théoriques et pratico-politiques du marxisme» (séance 9, p. 5). Quoi qu'il en soit, il est certain que la lecture de ces dernières séances laisse entrevoir la vertu singulière - peutêtre «cathartique», mais justement pas au nom d'un discours «purifiant» — de ce séminaire, du séminaire, pour Derrida. Et c'est bien de lecture qu'il s'agit. Avec et contre Marx, donc, ou ceux qui parlent en son nom.

32. «GREPH», séance 9, p. 4. 
$\mathrm{Au}$ contraire, "une lecture déclôturante, dé-limitante et militante ${ }^{33}$ » consisterait à souligner dans le texte de Marx les ruptures avec les schémas onto-téléologiques classiques, en particulier sous leur forme hégélienne. Derrida s'efforce alors de montrer que c'est précisément grâce aux notions offertes par le texte de Marx que nous pouvons faire ce «saut». Le texte de Marx serait donc déjà, partiellement mais irréductiblement, déconstructif ou auto-déconstructif. Le "saut» de l'Augenblick (l'instant d'un clin d'œil) est d'ailleurs un thème majeur et récurrent dans L'idéologie allemande, où il vient plusieurs fois perturber la course de la machine dialectique, déjouant l'Aufhebung pour l'entraîner ailleurs, d'un coup d'un seul, de manière aporétique mais avec la force irruptive, la coupure irréversible d'un événement. Derrida écrit: "Seule l'instantanéité peut donner sa forme - temporelle mais aussi intemporelle, l'instant étant à la fois temporel et intemporel, ou ni temporel ni intemporel — seul l'instant peut donner sa forme à ce saut ${ }^{34}$. » Il s'agit donc d'accompagner le saut de l'Augenblick, dans le cours du temps mais hors du temps, contre le temps et contre la dialectique reproductrice, en appuyant, par exemple, toutes les virtualités conceptuelles et les puissances métaphoriques que le texte de Marx offre à la lecture.

Derrida montre ainsi que la structure de l'idéologique suppose «une non-vérité structurelle » avec la division du travail «comme telle » ${ }^{35}$. Autrement dit, l'idéologie non seulement traduit ou trahit (en l'occultant partiellement) la division du travail, mais cette traduction produirait aussi une division asymétrique redoublant la division du travail, divisant la division et la rendant méconnaissable «comme telle». «Le travail travaille le travail» et «la division divise la division $»^{36}$. Derrida précise alors qu'il est difficile, bien sûr, de soutenir cette idée très loin en s'appuyant de manière conséquente sur les textes de Marx, puisque celui-ci pense aussi la division du travail comme un phénomène fini, et dépassable: «anticipation eschato-téléologique très nette d'une société sans division du travail ${ }^{37} »$. Ce serait ici, selon Derrida, la dimension hégélienne du projet marxiste, qui traverse la négativité pour aboutir à une réappropriation finale.

Néanmoins, Derrida souligne que, dès L'idéologie allemande, la position ou l'autoposition de la conscience effective (idéologique) est conçue par Marx comme secondaire, dérivée, comme résultant de rapports de force effectifs, d'une effectivité (Wirklichkeit) agonistique plus originaire, ce qui complique radicalement le schéma hégélien conventionnel de production de l'idéalité. L'idéologique étant toujours dérivé, il n'aurait pas d'origine pure. L'idéologique avait donc dû commencer dès le travail du Wirken, "avant la

33. Ibid., séance 9, p. 10.

34. Ibid., séance 9, p. 3.

35. Ibid., séance 9, p. 5.

36. Ibid., séance 9, p. 2.

37. Ibid., séance 9, p. 5-6. 
division du travail effective et persistera après la division du travail effective ». L'idéologique n'est donc pas seulement «un concept homogène ou le concept d'une chose homogène "; et on ne peut rêver "d'une abolition de l'idéologique, mais d'une transformation interminable de sa structure ${ }^{38}$.

Au cours de cette dernière séance, Derrida insiste sur le motif de la différence sexuelle, qui aura ainsi été l'un des «thèmes» majeurs du séminaire ${ }^{39}$. Derrida note que dans L'idéologie allemande la division du travail "effective" (la division entre travail matériel et travail intellectuel ou spirituel, geistig - celle qui produira l'idéologique «en tant que tel ») est, de fait, précédée d'une autre division du travail, plus primitive (ursprünglich): la division du travail dite «naturelle» (Marx utilise des guillemets) ${ }^{40}$. Or celleci «n'est pas autre chose que la division du travail dans l'acte sexuel ${ }^{41}{ }^{»}$. Derrida souligne cette étrange formule: die Teilung der Arbeit im Geschlechtsakt. Mais qu'est-ce donc que "l'acte sexuel comme travail ${ }^{42}$ »? S'agit-il, selon Marx, de «l'acte sexuel » proprement dit — le coï — ou de tout le dispositif de la reproduction sexuelle, la structure familiale hétérosexuelle et patriarcale, et la distribution de la différence sexuelle comme dualité des sexes?

38. Ibid., séance 9, p. 6.

39. Impossible d'évoquer tous les textes dans lesquels Derrida aborde la «question» de la différence sexuelle - ce motif court dans tous ses écrits, explicitement ou plus souterrainement. Je me bornerai à évoquer ici un texte plus ou moins contemporain de la période examinée: Éperons. Les styles de Nietzsche, Paris, Flammarion, 1978 (conférence donnée en 1972 lors de la décade de Cerisy Nietzsche aujourd'hui?, et dont une première version fut publiée en 1973 dans les actes du colloque). Pour le dire trop schématiquement, l'usage que fait Derrida de la notion de "différence sexuelle" renvoie non seulement à telle ou telle différence entre les sexes, leur répartition et distribution (notamment à travers les figures plus ou moins déterminées de «l'homme» et de «la femme », leur opposition duale voire leur réversibilité), mais aussi à une différance sans présence, rejouant sans cesse ces différences à travers des combinatoires toujours singulières, donnant ainsi lieu à des différences sexuelles indécidables et inassignables à des «corps" ou à des «sujets". Cette différance sexuelle se donne ainsi (à lire) sans être réductible à l'être ou à l'essence de ce qu'on nomme conventionnellement "sexe ", "sexualité » ou «gender» : «la différence sexuelle [n'est] pas une question régionale soumise à une ontologie générale, puis à une ontologie fondamentale, enfin à la question de la vérité de l'être ». Et Derrida ajoute: ce n'est "peut-être même plus une question» (Éperons, p. 89). Cette différence n'est donc ni une différence naturelle, biologique ou anthropologique, ni une différence "produite» (par exemple dans le sens de "production performative»). Elle excède d'avance la position d'un discours ou d'une question ontologique à son sujet. Pour une lecture de Derrida et Judith Butler à ce propos, voir l'essai de Peggy Kamuf, «Derrida and Gender: The Other Sexual Difference", dans Jacques Derrida and the Humanities, Tom Cohen (dir.), Cambridge University Press, Cambridge, 2001. Voir aussi Ginette Michaud, "Derrida and Sexual Difference ", dans, After Derrida: Literature, Theory and Criticism in the 21st Century, Jean-Michel Rabaté (dir.), Cambridge University Press, Cambridge, 2018.

40. Marx et Engels, L'idéologie allemande, p. 60. Cette axiomatique sexuelle est donc indexée sur une distinction nature/culture, animalité/humanité. Elle suppose une pensée anthropologiste de l'idéologique, et de la division du travail comme "propre " de l'homme, tendant à neutraliser du même coup les différences sexuelles et animales.

41. Ibid.

42. «GREPH», séance 9, p. 1. 
Quoi qu'il en soit, si la «division du travail naturelle» (sexuelle) et la «division du travail effective» (wirklich) partagent le même nom, il faut rendre compte de ce partage. La seconde division ne devient proprement «ellemême" qu'en se développant (entwickelt) à partir de la première. Ce développement (dit «naturel») permettra enfin le passage - le «temps» d'un Augenblick - de la société familio-patriarcale primitive à la société proprement dite.

Toutefois, la première division, sexuelle, dite " originaire ", ne peut pas ne pas se reproduire, se répéter par et dans la division du travail seconde, dite "effective " : c'est en effet l'essence de ladite "reproduction", qui présuppose ainsi du même coup une conception reproductrice de la famille, du sexe et de la sexualité. Certes, les deux divisions du travail sont séparées par l'événement de l'Augenblick, mais la division dite "naturelle» (sexuelle) reste requise par et dans la division du travail "effective» et l'organisation matérielle-idéologique (ou «idéologico-sexuelle») de la société. Cela implique que les deux divisions du travail puissent et doivent se contaminer l'une l'autre, et que la division du travail «effective» ainsi que les appareils idéologiques (par exemple l'École, l'Église, l'État, etc.) présupposent toujours l'autre différence, plus originaire, la sexuelle (ou «naturelle»). La division du travail est donc toujours-déjà sexuée, sexuelle ou sexuante. Et pourtant, la critique marxiste de l'idéologie tend à escamoter la structure familiale et la différence sexuelle en reproduisant une distinction privé/public (nature/ société) qui n'est pas elle-même pensée comme idéologique. Selon Marx, l'idéologique «en tant que tel» ne serait pas encore apparu avec la division du travail sexuelle et la structure familiale hétéro-patriarcale. Dans L'idéologie allemande, la division du travail "naturelle» (et, donc, sexuelle) ne relève pas de la division du travail «effective" ni ne relève de l'«idéologique», puisqu'elle précède l'émergence de l'idéologie (et de la société) «comme telle». Selon Derrida, cette distinction entre division «naturelle» (familiale-sexuelle) et division "effective» (sociale) tend à reproduire l'opposition privé/public, famille/société ou nature/culture que l'on retrouve chez Hegel, ainsi que chez tous les philosophes-économistes classiques (non marxistes) précités.

Et pourtant, donc, il y a, il y aura toujours eu division sexuelle. Et celle-ci reste la première, la plus "originaire", ursprünglich. La division du travail dite "effective» (wirklich) n'en est qu'un développement selon le processus de l'Entwicklung. Or, puisque la formation (Bildung) idéologique (ou l'imagination, Einbildung) trouve son origine dans la division du travail "effective", qui n'est elle-même qu'un développement de la division dite «naturelle» - et en premier lieu de l' «acte sexuel» (Geschlechtsakt, qui serait ainsi le premier travail, le premier Arbeit et le Wirken originaire) Derrida propose alors de penser l'idéologique non plus selon une trajectoire hégélienne, donc, mais dans la perspective psychanalytique du phantasme, d'un imaginaire (Einbilden) idéologique conçu comme "phantasmatisa- 
tion ». Toujours sur le mode d'une lecture déclôturante, Derrida saisit ainsi la problématique de l'idéologique chez Marx comme pensée de l'imagination phantasmatique, inconsciente, ou comme idéalité non consciente, «idéalité leurrée » — échappant ainsi, provisoirement du moins, aux chaînes de l'hégélianisme:

Cela voudrait dire qu'avant la conscience idéologique ou la représentation idéologique, il y a une idéalité leurrée non consciente. Que Marx l'ait reliée à la famille et à la division du travail naturelle dans l'acte sexuel, qu'il ait lié l'idéologique pré-conscient à la différence sexuelle, voilà qui pour le coup peut paraître beaucoup plus inédit et singulier. Bien sûr, Hegel aussi a lié l'idéalisation à la différence et à la différence sexuelle mais il n'a pas — semble-t-il — comme Marx inscrit le leurre irréductible dans le procès d'idéalisation. Il n'a pas soustrait la phantasmatisation, de façon irréductible et structurelle, au mouvement de la vérité et de la présence ou de la conscience de soi, comme Marx tel du moins que je le lis ici ${ }^{43}$.

Puisqu'ici sexe et sexualité sont pensés comme travail et comme division comme Wirken, travail effectif - il devient difficile de leur assigner une origine et une fin. L'acte sexuel (Geschlechtsakt) se dédouble, se divise dans le mouvement dialectique, et dans l'idéologique qui en est le produit. Cela témoigne de la difficulté de réduire la «question» sexuelle, de la contenir dans des limites stables. Cela suppose aussi le débordement d'oppositions telles que nature/culture ou famille/société. Si «l'idéologique» trouve son origine dans la division sexuelle, il doit être pensé comme l'effet de processus psychosexuels, effet de sexe, du sexe ou des sexes, de la différence ou des différences sexuelles telles qu'elles s'écrivent et se lisent, se traduisent à travers l'idéologique. Celui-ci se voit donc irréductiblement affecté par les différences de forces et les divisions agonistiques dont il est le produit, à commencer par la division dite "sexuelle", qui en serait l'origine. Cela exige du même coup une prise en considération du caractère dynamique de la division (sexuelle), de son mouvement comme différentiel de forces, comme «différance agonistique» — sans possibilité de la stabiliser ou de la réduire sous la forme (ontologique) d'une réappropriation, d'une simple «reproduction ", ou d'une relève dialectique (Aufhebung):

43. Ibid., séance 9, p. 9. Environ deux ans plus tard, dans le séminaire "Théorie et pratique» (1976-1977), Derrida fera référence à toute cette analyse dans les termes suivants: "Or dans la mesure où la pratique sexuelle, en tant qu'elle engage, et engage un corps qui ne se résume pas aux sens dits théoriques, la vue et l'ouïe, opposés aux sens que Hegel appelle pratiques [...], dans la mesure, dis-je, où la pratique sexuelle n'est pas un exemple parmi d'autres, vous pressentez que la question du travail et de la division du travail, de la division sexuelle du travail dans la problématique marxiste, cet ensemble de questions que nous avions abordées ensemble il y a deux ans ici et que nous retrouverons encore, est bien un ensemble systématique et non localisable comme ensemble de questions particulières et dérivables. » Derrida, Théorie et pratique, p. 50. L'éditeur identifie le séminaire en question comme étant «L'art (Kant)» — mais il s'agit bien sûr de celui dont nous parlons ici. 
Lier le procès idéologique - avant même sa conscience et son effectivité - à la différence sexuelle, c'est non seulement impliquer que la phantasmatisation (l'Einbildung) n'est pas une imagination du type classique de la représentation consciente en marche vers la vérité, mais c'est la lier à une différence de force dans des rapports de domination et dans un espace de contradiction, ou en tout cas de différance agonistique. Déjà dans ce qu'il [Marx] appelle la division du travail naturelle (division du travail sexuelle, etc.), la division du travail se fait en fonction de différences de forces (par exemple Körperkraft). L'idéalité, en tant que liée à une lutte mettant en jeu des forces et visant à la maîtrise, devient elle-même, est elle-même un pouvoir tendant à la domination (d'où d'ailleurs la difficulté à distinguer entre le matériel et le spirituel: une technique est déjà une idéalité, etc.) si bien qu'on ne peut pas penser le procès idéologique sans lutte pour la puissance, sans tendance de l'idéologie à devenir dominante (la dominance n'est pas un caractère qui survient à l'idéologie, c'est sa loi tendancielle) et sans rapport à la différence sexuelle qui est sinon l'origine réelle du moins le point zéro à partir duquel on peut penser quelque chose comme la division du travail et l'idéologie. Dans ces conditions, tout ce que Marx dira dans les pages suivantes sur le rapport entre l'idéologie effective et les contradictions dans les rapports de force économiques doit avoir, au moins en principe, un lien essentiel, jamais rompu, avec la différence sexuelle, avec l'origine structurelle du travail et de la division du travail ${ }^{44}$.

En insistant sur l'idéologique comme phantasmatisation - de type psychosexuel, donc - Marx, tel que lu ici par Derrida, aurait ouvert la voie à la pensée d'une différance agonistique, "originaire » avant l'origine, un différentiel de forces supposant frayage, répression et refoulement, et signalant du même coup un certain secret de l'idéologique inséparable de l'organisation idéologico-sexuelle du socius. Cette différance agonistique est ce qui à la fois limite et ouvre le champ de la lecture - un champ sexuel, donc, et une lecture sexuée. On pourrait lier tout cela à ce que Derrida écrira plus tard dans "Fourmis" au sujet de la différence sexuelle comme fable, comme fabuleuse ou fabulaire, et sur le caractère irréductiblement sexué de la lecture:

Si la différence sexuelle est toujours lue, elle est aussi lisante, c'est-à-dire qu'elle est lue, comme différence sexuelle, dans et par la différence sexuelle, à travers elle [...]. Double génitif de l'expression «lecture de la différence sexuelle». Cela signifie qu'il n'y a pas de lecture asexuelle, asexuée ou métasexuée de la différence sexuelle puisque celle-ci est à la fois lue et lisante. [...] elle lit, la différence sexuelle, autant qu'elle est lue, il n'y a pas de lecture asexuée ou métasexuelle ${ }^{45}$.

La différence sexuelle (lisante et lue) espace donc le discours ontologique, espace le présent, et le temps, le temps de la lecture: "Il n'y a pas de rendez-

44. «GREPH», séance 9, p. 9-10.

45. Jacques Derrida, "Fourmis", dans Lectures de la différence sexuelle, Mara Negrón (dir.), Des Femmes, Paris, 1994, p. 86-87. 
vous sans l'espace du contretemps, sans l'espacement du contretemps, et pas de contretemps sans différence sexuelle, comme si la différence sexuelle était le contretemps même ${ }^{46} »$. On retrouve ainsi le thème de la lecture - lecture en cours: le cours comme lecture, ou ce qui s'appelle lecture en anglais que nous n'avons bien sûr jamais quitté. Il s'agirait ici d'une lecture "déchaînée » de Marx, que Derrida souhaite associer (ou accoupler) dans le cours de 1974-1975, de manière certes violente et anachronique, mais productrice et émancipatrice, à une certaine problématique freudienne: «déchaîner» le texte, c'est l' "articuler avec une problématique elle-même compliquée de type psychanalytique articulant la question de l'idéologie sur celle du phantasme, de l'inconscient, etc. ${ }^{47}$ » Avec Marx et sa pensée de "l'idéologique ", un "garde-fou » a sauté. Marx nous autorise à interroger une limite, "celle de la vérité ou du sens caché dans un texte offert à une lecture simplement théorique ou observatrice ${ }^{48}$ ». La vérité se voit prise dans la promesse d'une lecture à venir, promesse d'émancipation, d'une transformation émancipatrice qui interdit de réduire la vérité à un simple dévoilement ou à une simple explicitation, à la reproduction d'un contenu déjà donné. Ça déborde, la véritée ${ }^{49}$. Et ce qui fait cela à la vérité, c'est précisément le texte - ce que Derrida nomme «le texte-Marx» :

Le texte de Marx, le texte-Marx a contribué à cet ébranlement qui nous a permis - avec Freud aussi bien, Nietzsche et quelques autres qui sont comme ceux-là des effets autant que des auteurs - de pratiquer des lectures transformatrices, impliquant des interventions militantes et actives, écrivant un autre texte en lisant, etc. Il va de soi qu'à partir du moment où cette possibilité appelons-la encore historique - est ouverte, on ne peut plus enseigner de la même manière. L'enseignement, la pratique enseignante est, doit être en tous

46. Ibid., p. 80. Ce contretemps et cet espacement sont aussi la marque d'une différence sexuelle qui ne s'appartient jamais en propre. Pour un déploiement vertigineux de motifs «sextuels» que je ne peux malheureusement explorer ici, mais dont les traces s'offrent pourtant déjà à une lecture disséminatrice (peut-être, et certainement, malgré moi), je renvoie à l'essai « séminal » de Peggy Kamuf, "Derrida and Gender»: "That probability is even a certainty if one takes as seriously as one should dissemination as Derrida has described it and which he understands to be at work in every text as its possibility, that is, the possibility of its coming to find a reader. This term can still be heard in its so-called sexual (that is, genital) sense: a dis-semination, where the prefix has a privative value. It would be a non-semination, a non-generative nonreproduction of the seed and the semen, which is the masculine essence. But the word has not just a privative sense, because it also says dissemination, the scattering of the semantic or semiotic value of signs. Between these levels, between the body of signs and the genitally sexed body, the act of semination is itself dis-seminated, meaning it does not reproduce itself, no "itself" can reproduce itself» (p. 88, c'est Kamuf qui souligne). - Et plus loin elle écrit, la différence sexuelle, elle s'écrit: "Sexual difference divides - itself. It thus divides its proper meaning and this division inscribes (itself as) a text» (p. 102).

47. "GREPH », séance 9, p. 10.

48. Ibid.

49. Voir Derrida, Théorie et pratique, p. 121: «S'il y a débordement, effet de débordement, c'est un effet de vérité. C'est au nom de la vérité que ça déborde toujours. » 
cas bouleversée. On ne peut plus apprendre à lire comme auparavant, croire ou faire semblant de croire que lire c'était dévoiler la vérité d'un texte, cachée et univoquement contenue dans un texte qu'il suffit de répéter ou de reproduire. Pour boucler ce que je disais au début de ce séminaire du répétiteur, l'enseignement ne peut plus être reproducteur et répétiteur, fût-ce du texte le plus révolutionnaire, fût-ce du texte-Marx. Enseigner Marx ou tous les textes de l'époque dont je parle, cela ne peut plus se faire par reproduction ou répétition ou dévoilement ou explicitation. En ce sens la question de l'enseignement n'est pas une question sur laquelle Marx a pu se prononcer, sur laquelle nous avons à chercher chez lui ou chez d'autres des énoncés reproductibles. La question de l'enseignement est pratiquement posée et ne peut être que pratiquement résolue par la transformation de l'enseignement, de l'appareil et des procédures d'enseignement à laquelle ces textes - et vous savez quelle extension je donne à ce mot de texte - nous contraignent. Nous traitons de l'enseignement en le transformant, c'est à dire, par exemple, en lisant autrement, en déconstruisant les modèles de lecture ou de transmission, la lecture et la transmission et la répétition sur lesquelles était construit l'enseignement dominant $t^{50}$.

La pensée du discours philosophique comme texte bouleverse donc le "concept» de l'idéologie, y compris sous sa forme critique, et le rapport de la philosophie à son inscription matérielle — c'est-à-dire aussi la pratique enseignante. La pratique du lecteur, du répétiteur, non seulement " reproduit », mais doit aussi conduire ou reconduire la possibilité de l'interprétation transformatrice et de la lecture disséminatrice, la dissémination comme possibilité de la lecture. L'idéologique n'est plus simplement expression (Ausdruck) d'une source (pratique ou matérielle) préexistante: le médium — texte, cours, lecture - est déjà écriture. De même, l'enseignement n'est plus, ne peut plus être conçu comme simple répétition-reproduction, «fût-ce du texte le plus révolutionnaire, fût-ce du texte-Marx». Derrida ne pourrait être plus clair : la lecture révolutionne la révolution. La lecture, c'est l'avenir. Lire Marx, c'est lui être infidèle par fidélité, et ainsi l'enseigner selon des modalités qui transforment les rapports de l'enseignant à son propre cours, et les rapports de l'enseignement de la philosophie à ce qui constitue un discours ou un texte "philosophique».

Il s'agit donc, en lisant, de repenser à nouveaux frais ce que «lire » ou «donner un cours» veut dire. Penser cela, c'est dire que l'enseignant est toujours débordé. Débordé, car la lecture en cours se donne pour l'autre. Dès lors, le cours se déborde, est d'avance débordé dans sa course, entraîné vers l'autre rive, la rive de l'autre - par le texte. Ça déborde. Cela arrive dès l'adresse, dès avant le cours «comme tel ». D'avance la course s'interrompt, mais elle vit de cette interruption, qui l'entraîne ailleurs et la relance déjà. Il faut courir. Le cours s'envoie, se donne et se confie à l'autre avant d'être lui- 
même, "proprement» lui-même. Le cours s'appelle déjà, dérive — à la dérive depuis la réponse de l'autre.

Certes, cette dérivation du cours en cours signe d'avance la défaite du Maître, sa finitude et celle de son discours, leur essoufflement, voire leur épuisement. Mais c'est aussi, si on l'affirme, une source infinie de surprise, et de plaisir:

Si bien que je ne suis jamais au bout de mes surprises quand je fais un cours. La surprise est cette structure qui replie le métalangage, en surprend toujours la niaiserie $[. .$.$] et procure, pourvu que cette surprise soit affirmée et réaffirmée$ par qui a la force d'y céder enfin, le plaisir, un plaisir qui doit être, comme il appartient à l'essence du plaisir ou du désir, qui doit être et rester douteux, confié à l'autre comme à prendre ou à laisser. On ne sait jamais où va un cours. Ce dont on parle et ceux à qui l'on parle ont toujours en réserve de quoi dire d'avance non seulement sur le cours, mais sur la théorie pédagogique ${ }^{51}$. 